\title{
Visual thinking como nexo entre Ciencia y Arte
}

\section{Laura Osete Cortina ${ }^{a}$ y Carla Álvarez Romero ${ }^{b}$}

anstituto Universitario de Restauración del Patrimonio. Universitat Politècnica de València, Camino de Vera s/n, 46022, València. e-mail:losete@crbc.upv.es. 'Instituto Universitario de Restauración del Patrimonio. Universitat Politècnica de València, Camino de Vera s/n, 46022, València. e-mail: carla.alvarez.romero@gmail.com.

\begin{abstract}
In this work, visual thinking is proposed with the aimed of facilitating the learning of scientific-technic subjects aimed at students with a humanistic profile. Scientific concepts of great difficulty are more easily comprehended throughout the expression of ideas in a graphic way. For that reason, the use of the artistic language, common in the field of Fine Arts is planned, for the purpose of the scientific study of artworks by means of collaborative activities.
\end{abstract}

Keywords: visual thinking, restoration, instrumental techniques, works of art, collaborative learning.

\begin{abstract}
Resumen
En este trabajo, se propone la aplicación de la herramienta visual thinking con la finalidad de facilitar el aprendizaje de disciplinas científico-técnicas a alumnos de formación humanistica. Los conceptos científicos de gran dificultad son más fácilmente asimilables mediante la expresión de ideas y pensamientos de forma gráfica. Para ello se plantea el uso del lenguaje artístico, común en el ámbito de las Bellas Artes, al servicio del análisis científico de obras de arte, a través de actividades colaborativas.
\end{abstract}

Palabras clave: visual thinking, restauración, técnicas instrumentales, obras de arte, aprendizaje colaborativo.

\section{Introducción}

Dentro del máster de Conservación y Restauración de Bienes Culturales ofertado por la Facultad de Bellas Artes de la Universitat Politècnica de València (UPV), se encuentra la asignatura optativa "Técnicas Instrumentales para el desarrollo de la experimentación en la Conservación y Restauración del Patrimonio". Esta asignatura, se centra en la revisión y el acercamiento al estudio científico del Patrimonio Cultural desde el punto de vista de las Técnicas Experimentales. La investigación de materiales, técnicas artísticas, alteraciones y productos empleados en la conservación y restauración requieren un estudio exhaustivo desde diversos enfoques complementarios. Normas, ensayos e instrumentación y técnicas de análisis son las herramientas de las que dispone el investigador del Patrimonio para contestar 
de manera objetiva a las múltiples cuestiones que se plantean ante los tratamientos conservativos-restaurativos de un objeto artístico o/y arqueológico.

Al tratarse de una asignatura optativa de segundo curso de Máster, el grupo de alumnos matriculados es reducido, en concreto 14 alumnos, que suponen el 50\% del total de matriculados en el Título. Como aspecto a destacar, hay que mencionar, que el carácter altamente técnico de esta asignatura choca abruptamente con el perfil humanístico (restauradores) de los estudiantes a los que va dirigida, que carecen de una base científica sólida. A esta carencia hay que añadir, además, la dificultad que implica que los alumnos han de familiarizarse con un lenguaje científico muy específico que dificulta el aprendizaje significativo de estos nuevos conceptos. Partiendo de este hándicap, se plantea la necesidad de aplicar metodologías de enseñanza-aprendizaje que tengan en cuenta el perfil académico de los alumnos, las características de la formación en competencias, los conocimientos actuales sobre los procesos de aprendizaje eficaz, y que permitan el acercamiento del mundo científico con el artístico mediante un lenguaje común que facilite la comprensión e integración. En este sentido, el pensamiento visual o visual thinking destaca entre otras herramientas de aprendizaje disponibles al estar basada en el uso de estrategias y técnicas empleando un lenguaje visual en la transmisión, compresión o asimilación de conceptos (Glaser, 2008; Roam, 2010). Estas características hacen que, a priori, pueda actuar como nexo de unión entre una disciplina científica y el ámbito artístico, facilitando de esta manera el aprendizaje.

\section{Objetivos}

El principal objetivo de la actividad que se plantea es establecer metodologías para facilitar el aprendizaje de conocimientos y terminología científica en el ámbito de las Humanidades. Se pretende, personalizar la enseñanza al perfil de los alumnos, fomentando que la creatividad, que de manera innata disponen la mayoría de ellos al pertenecer al ámbito artístico, se ponga al servicio del aprendizaje activo. Para ello, se plantea la realización de una actividad basada en la herramienta visual thinking, que permita:

- Integrar nuevos conocimientos científicos mediante el uso de un lenguaje artístico, con la finalidad de facilitar un aprendizaje significativo.

- Interiorizar, explicar y compartir ideas en actividades de tipo colaborativo. 


\section{Desarrollo de la innovación}

En el marco del Proyecto de Docencia Inversa de la UPV, en la asignatura de "Técnicas instrumentales para el desarrollo de la experimentación en Conservación y Restauración del Patrimonio", se sigue un modelo pedagógico que transfiere fuera del aula el trabajo de determinados procesos de aprendizaje que implican el alcance de habilidades de nivel cognitivo inferior (recordar o comprender), mientras que se emplea el tiempo de clase a través del apoyo docente, para facilitar y potenciar otros procesos de adquisición y práctica de conocimientos dentro del aula, dirigidos a alcanzar habilidades de nivel cognitivo de mayor complejidad (aplicar con cambios, analizar, evaluar, crear) (Tourón y Santiago, 2015). De esta manera, partiendo de los resultados de aprendizaje a alcanzar por el alumno, se elaboran los contenidos de la asignatura, y se diseñan las actividades a realizar tanto fuera como dentro del aula. En concreto, para la unidad didáctica "Técnicas de análisis instrumental en Patrimonio", donde se tratan conceptos básicos sobre las principales técnicas de análisis científico como fundamentos, instrumentación, información analítica que proporcionan y aplicaciones en Patrimonio, se plantean como resultados de aprendizaje:

- Distinguir y estructurar los aspectos más relevantes de las técnicas instrumentales más empleadas en el estudio científico del Patrimonio Cultural

- Comparar dichas técnicas y sus aplicaciones entre sí, dentro del ámbito de los bienes culturales.

Con el propósito de alcanzar estos resultados de aprendizaje, se diseñó una actividad colaborativa basada en las técnicas de visual thinking y puzzle de Aronson (Domingo, 2008; Ramil y Rodríguez, 2012). Para todas las actividades colaborativas realizadas en el aula se crearon grupos de cinco participantes. La actividad previa al trabajo en el aula consistió en la visualización de un video screencast sobre una de las técnicas instrumentales de análisis empleadas en Conservación y Restauración del Patrimonio, que posteriormente, el alumno tendría que comentar y compartir con el resto de los miembros de su grupo, que habrían trabajado sobre el resto de las técnicas de análisis hasta un total de cinco. A cada uno de los participantes del equipo se le asignó una técnica analítica diferente, y la elaboración de un mapa mental individual en el que plasmar de manera visual las características principales presentadas en el video. Los contenidos que debían representar fueron los aspectos más relevantes de cada técnica, como fundamentos, equipamiento empleado, información que proporciona sobre el objeto cultural, así como las principales aplicaciones en dicho ámbito. Posteriormente, en el aula, la actividad grupal se basó en el desarrollo de un puzzle de Aronson, en el que, en una primera fase de expertos, se reunían todos los alumnos que habían trabajado sobre una misma técnica de análisis, y posteriormente, se debía realizar una puesta en común de cada participante en su propio grupo exponiendo el mapa mental realizado de manera individual y las aportaciones del resto de compañeros de la fase previa de expertos. De esta manera, todos los miembros del equipo se pudieron familiarizar con el resto de las 
técnicas y elaborar un mapa mental grupal en el que se integraran y compararan las cinco técnicas de análisis más relevantes para el estudio del Patrimonio. Una última fase consistió en la exposición por parte de cada grupo del mapa mental grupal elaborado.

Aunque la duración del trabajo previo individual de cada alumno para la realización del mapa mental se estimó de una hora, mediante otras actividades grupales realizadas en sesiones previas, como estudio de casos, se habían trabajado también aspectos generales de las técnicas de análisis y sus aplicaciones en el ámbito de la Conservación del Patrimonio. Para el desarrollo de la actividad en el aula se empleó una sesión de dos horas. La temporización de la actividad se puede observar en la Figura 1.

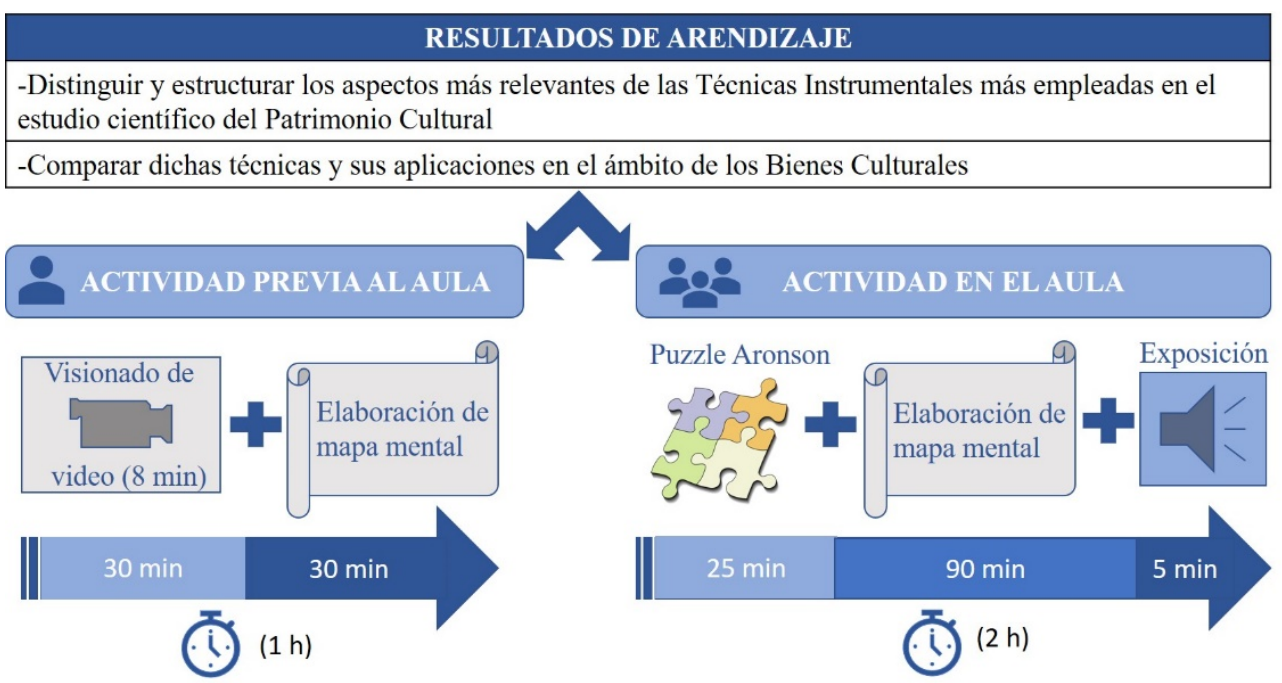

Fig. 1. Diseño de las actividades fuera y en el aula

\section{Resultados}

Los principales resultados a destacar de la actividad previa a la sesión del aula, consistente en la realización del mapa mental individual, fueron la gran implicación de los alumnos en la realización de la actividad. Como puede comprobarse, al observar como ejemplo uno de los mapas mentales realizado por una alumna (Fig. 2), destaca el elevado grado de creatividad empleado por los alumnos para plasmar unos aspectos tan técnicos como son las principales características de la Microscopía Electrónica de Barrido con microanálisis de rayos-X (SEM/EDX) aplicada al examen científico de los Bienes Culturales. Asimismo, no sólo se observó un mayor grado de implicación, lo que se traduce en un aprendizaje más activo, sino una adaptación del lenguaje científico al artístico, eliminando la barrera inicial debida a su formación humanística, lo que permite procesar cognitivamente la nueva información favoreciéndose el aprendizaje significativo a través de la representación gráfica y conceptual. La mejora en la comprensión e integración de estos nuevos conceptos quedó patente, además, 
en la primera fase de la actividad realizada en el aula, basada en el puzzle de Aronson, cuando cada alumno tenía que explicar a sus compañeros de grupo el mapa mental individual realizado, después de la fase de expertos.
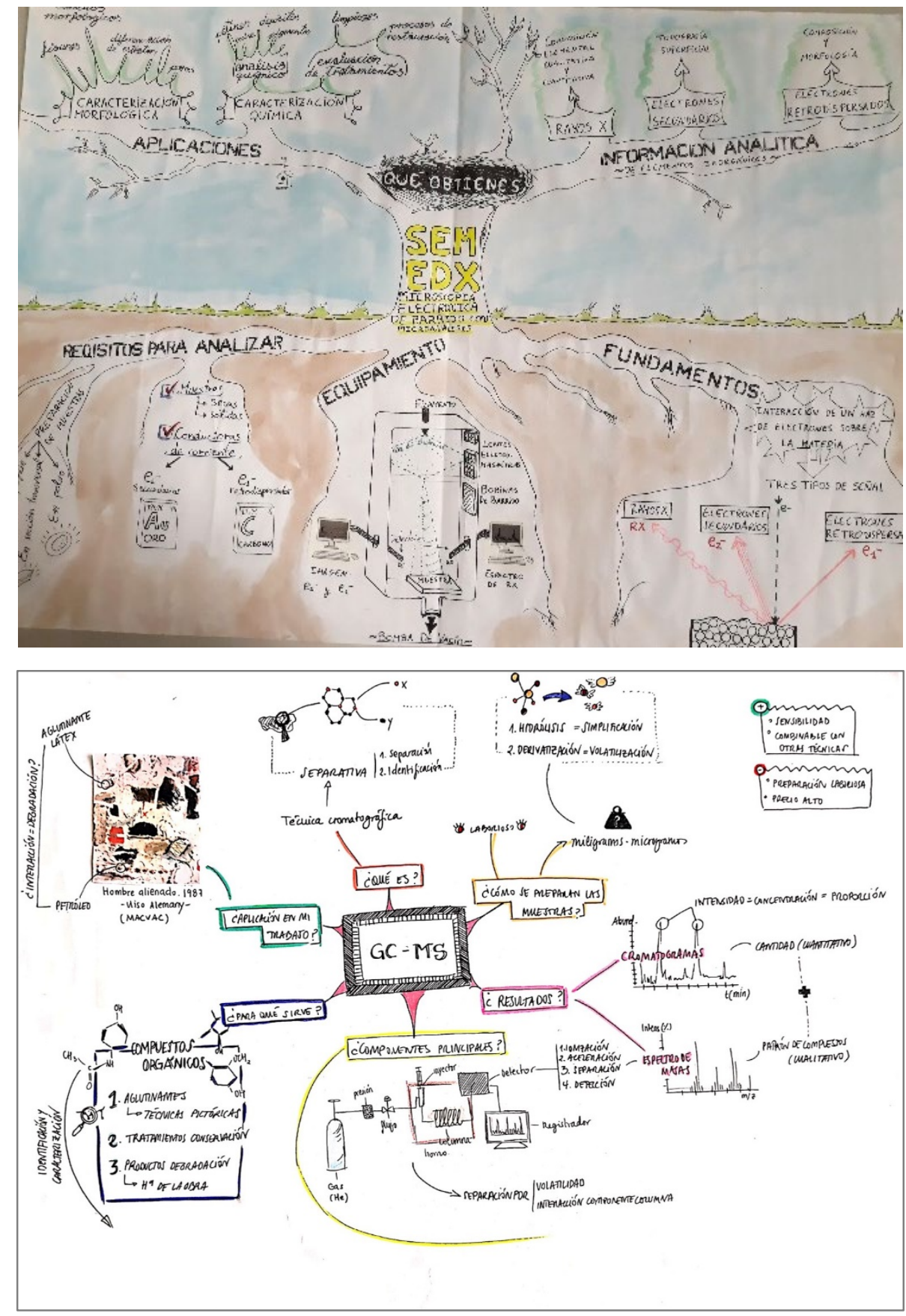

Fig. 2. Mapas mentales individuales sobre las principales características de las técnicas de análisis SEM/EDXy $G C-M S$ 
Con el propósito de evaluar este tipo de actividad, que presenta un importante componente subjetivo, se elaboró una rúbrica, en la que se consideraron los siguientes criterios, que, además, presentaban un peso variable en la calificación final:

- Capacidad de síntesis de los principales aspectos que caracterizan las técnicas de análisis estudiadas $(50 \%$ del peso)

- Exposición oral del mapa mental, valorándose el grado de dominio del tema a partir del uso de un lenguaje técnico apropiado, un discurso organizado y argumentación coherente $(25 \%$ del peso)

- Exposición creativa de los contenidos que se refleja también por el grado de implicación del estudiante en el producto final (15\% del peso total)

- Aportaciones personales, en relación con contribuciones adicionales del alumno en relación a su propio trabajo de investigación ( $10 \%$ del peso total)

A continuación, se muestra la rúbrica desarrollada.

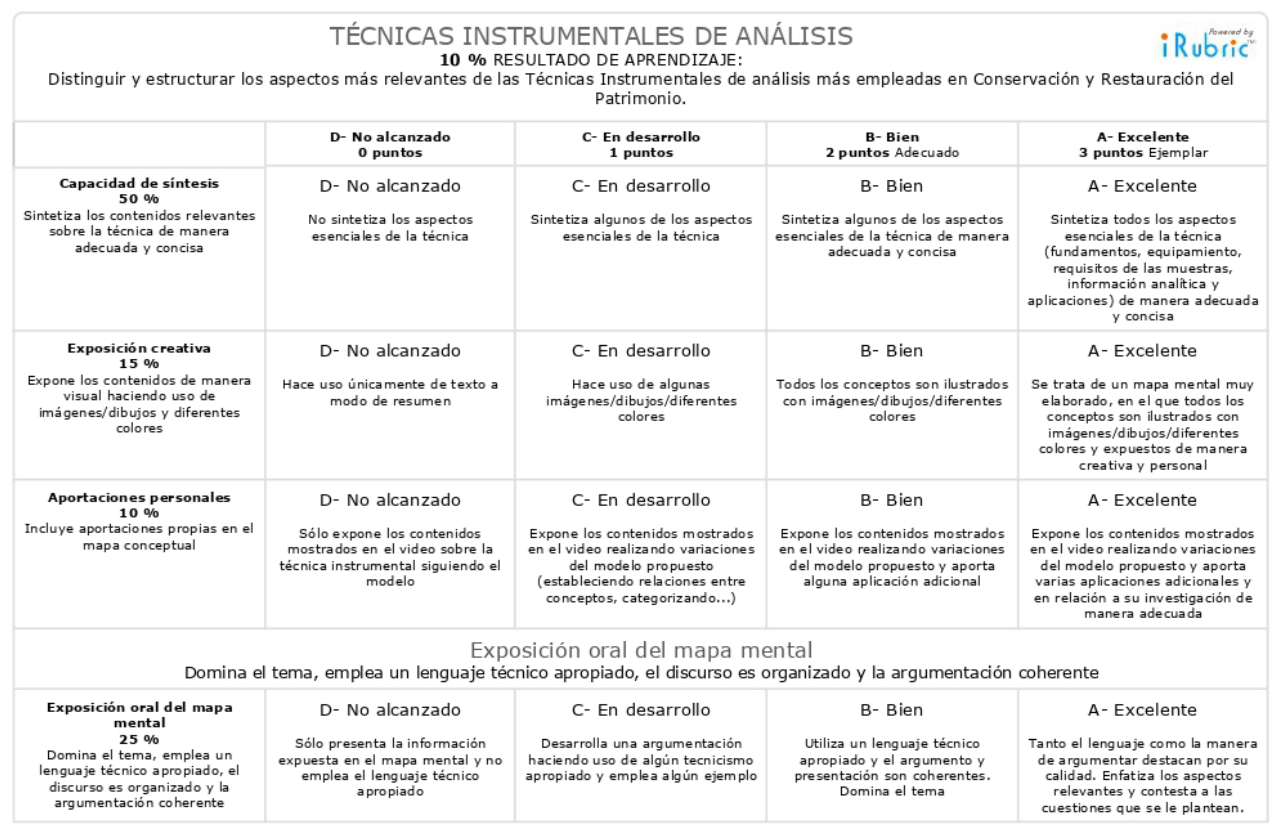

Fig. 3. Rúbrica desarrollada para la evaluación del mapa mental 
Otro aspecto importante a mencionar de esta actividad, fue el elevado nivel de motivación observado en los alumnos, hecho que pudiera estar relacionado con la integración de aspectos propios tan desarrollados como su creatividad. Por otro lado, en comparación con el grado de implicación general de los alumnos en el desarrollo de la actividad propuesta, con respecto a otras actividades grupales realizadas en el aula que no se basaban en la herramienta de visual thinking, se observó una mayor satisfacción general e implicación, resultado que se evidenció además en los comentarios de los alumnos plasmados en encuestas realizadas por el ICE de la UPV para la valoración de las actividades realizadas en la asignatura, así como en encuestas internas realizadas por las profesoras al finalizar el curso. En ellas, los alumnos destacaron su satisfacción por la realización de esta actividad e indicaron, por ejemplo, "su utilidad en la comprensión de las técnicas de análisis" y "que reforzaba el aprendizaje por la asimilación instantánea de conceptos complejos y la comprensión automática de nociones básicas mediante técnicas visuales".

Por otro lado, consideramos que el diseño de la actividad propuesta, que requiere la participación de todos los alumnos del grupo para la consecución del objetivo final de la creación de mapa conceptual grupal, asegura el trabajo en equipo y la mayor implicación de todos los participantes. La segunda fase de la actividad, consistente en la elaboración del mapa metal grupal y la exposición final del mismo a la clase, permite trabajar también aspectos de un mayor nivel cognitivo como el análisis y la síntesis, y competencias transversales como la comunicación efectiva. En la siguiente figura (Fig.4) se muestra como ejemplo uno de los mapas mentales grupales realizados por los alumnos.

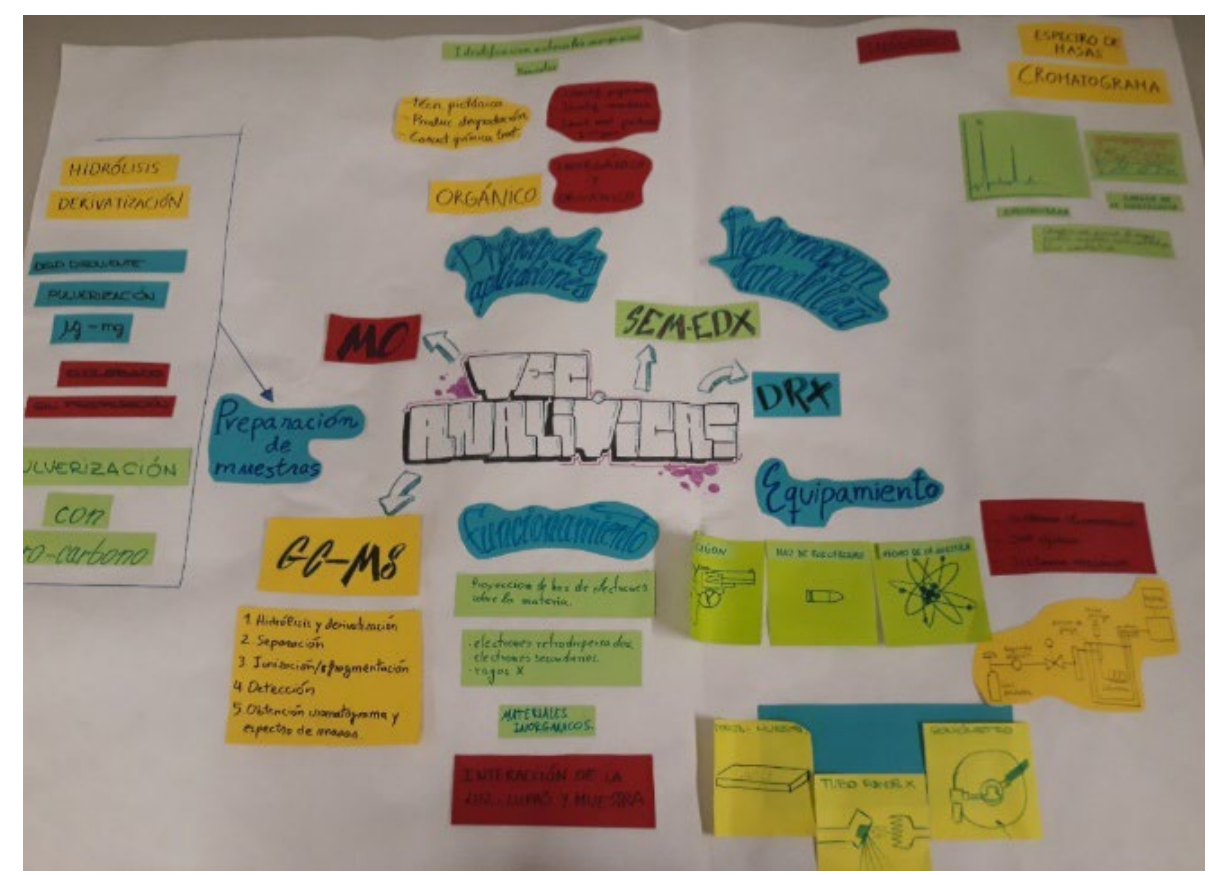

Fig. 4. Mapa mental grupal sobre las principales características de las técnicas de análisis más empleadas en el examen cientifico de Bienes Culturales 
Hay que destacar también, que teniendo en cuenta el modelo de formación por competencias, con la realización de esta actividad, se está potenciando el desarrollo de competencias transversales como la comprensión e integración, el trabajo en equipo y la comunicación efectiva, favoreciendo de esta manera la inserción en el mundo laboral de los alumnos en un futuro.

\section{Conclusiones}

La aplicación de herramientas de aprendizaje basadas en visual thinking dentro de actividades colaborativas en el aula constituyen un medio eficaz para favorecer la asimilación de nuevos conocimientos de una manera divertida, que incrementa la motivación de los alumnos y el grado de implicación en su proceso de aprendizaje.

En el contexto específico de la enseñanza de asignaturas de carácter científico a alumnos con una formación humanística, este tipo de actividades además intervienen en la creación de un ambiente de aprendizaje positivo, en el que conceptos complejos de carácter científicotécnico se traducen a un lenguaje visual más afín al perfil artístico de los alumnos. Asimismo, los elementos propios de la actividad basada en el puzle de Aronson favorecen el trabajo en equipo, asegurando la participación individual de todos los participantes.

\section{Referencias}

DOMINGO, J. (2008): “Aprendizaje cooperativo” en Cuadernos de trabajo social, 21, pp. 231-246.

GLASER, M. (2008): Drawing is thinking. Nueva York: Harry N.Abrams.

RAMIL CRIADO, M.; RODRIGUEZ PEREIRO, I. (2012): “Aprendizaje cooperativo en la universidad: ejemplo de aplicación de la técnica puzzle de Aronson a la enseñanza de Química Analítica" en Membiela Iglesia, P.; Casado Bailón, N.; Cebreiros Iglesias, M.I. Investigaciones sobre docencia universitaria y nuevas metodologías, pp. 267-270.

ROAM, D. (2010): Tu mundo en una servilleta. Málaga: Agapea Libros.

TOURÓN, J. y SANTIAGO, R.E. (2015): "El modelo Flipped Learning y el desarrollo del talento en la escuela” en Revista de Educación, 368, pp. 209-225. 